Volume 2

Issue 4 -- Integrative Medicine

Article 20

$11-20-2015$

\title{
Delirium Recognition in Hospitalized Older Patients: A Quality Improvement Project
}

Jodi Punke

Ariba Khan

Michael L. Malone

Follow this and additional works at: https://aah.org/jpcrr

Part of the Geriatrics Commons, and the Medical Education Commons

\section{Recommended Citation}

Punke J, Khan A, Malone ML. Delirium Recognition in Hospitalized Older Patients: A Quality Improvement Project. J Patient Cent Res Rev 2015;2:208. http://dx.doi.org/10.17294/2330-0698.1230

Published quarterly by Midwest-based health system Advocate Aurora Health and indexed in PubMed Central, the Journal of Patient-Centered Research and Reviews (JPCRR) is an open access, peer-reviewed medical journal focused on disseminating scholarly works devoted to improving patient-centered care practices, health outcomes, and the patient experience. 
oncogene that is overexpressed in $25-30 \%$ of breast cancers. In combination with first-line therapy, trastuzumab resulted in significant improvement in survival outcomes for those with HER2-positive metastatic breast cancer. Due to its improvement in outcome and prolonged survival, trastuzumab has been established as standard of care in both adjuvant and metastatic settings. However, along with common adverse events, trastuzumab has been found to be associated with cardiotoxicity. An estimated $1-4 \%$ of patients treated with trastuzumab will develop heart failure and $\sim 10 \%$ of patients will experience a reduction in left ventricular ejection fraction (LVEF). Many studies have published on the risk factors of trastuzumab-induced cardiotoxicity (TIC), with some discrepancy. Whereas one study found that of all risk factors accounted for (age, hypertension, LVEF, radiotherapy) only age was significantly associated with TIC, another found that LVEF was the sole factor, and others found that a combination of these were indicative of TIC.

Purpose: This paper aims to consolidate the data and identify potential risk factors from combined data.

Methods: A computer-based literature search using MEDLINE database was executed using the keywords trastuzumab/Herceptin, risk factors, outcomes, cardiac, cardiotoxicity, cardiomyopathy, LVEF and chemotherapy. Only prospective/retrospective human studies were included, with additional studies excluded if they reported a baseline LVEF $>68$, a cohort $<50$ patients, and/or results were not stratified based on cadiotoxic events.

Results: Data was collected from 17 articles, capturing 6,527 patients. A familial history of cardiac disease (odds ratio [OR]: $3.31,95 \%$ confidence interval [CI]: 1.80-6.08; $\mathrm{P}<0.01$ ), diagnoses of hypertension (OR: $1.61,95 \% \mathrm{CI}$ : 1.14-2.26; $\mathrm{P}<0.01$ ), diabetes (OR: 1.62, 95\% CI: 1.1-2.38; $\mathrm{P}=0.014$ ), and previous anthracycline use (OR: 2.14, 95\% CI: $1.17-3.92 ; \mathrm{P}=0.013)$ were all shown to be associated with TIC. Age $(\mathrm{P}=0.013)$ also was a risk factor.

Conclusion: Additional measures need to be set in place for monitoring cardiac performance in women treated with trastuzumab. Being aware of the potential risk factors along with careful attention to symptoms/LVEF can hopefully minimize the occurrence of TIC in this population.

\section{Delirium Recognition in Hospitalized Older Patients: A Quality Improvement Project}

\section{Jodi Punke, Ariba Khan, Michael L. Malone}

\section{Department of Geriatrics, Aurora Sinai Medical Center and Aurora UW Medical Group}

Background: We noted a low reported prevalence of delirium $(3 \%)$ in hospitalized older patients at a community teaching hospital in north central Wisconsin.

Purpose: This was a quality improvement project to report recognition of delirium by nurses before and after an educational intervention.

Methods: This project was performed on one medical unit in our hospital. Quality improvement data was collected at baseline and after the educational intervention. Data collected included observation by a geriatrician attending weekly interdisciplinary rounds to note any mention by nurses of delirium or confusion. The patient's electronic health record (EHR) was reviewed to note delirium assessment by "confusion assessment method for the intensive care unit (Vanderbilt)" (CAM-ICU) by the nurses for 2 days prior to the team meeting. The numbers of positive and total attempted CAMICU were recorded. Use of antipsychotics or benzodiazepines was reported as a "delirium marker." Diagnosis of delirium and dementia was obtained from the problem list in the EHR. The educational intervention included Just-in-Time Teaching during weekly Acute Care for Elders rounds during a 1-month period.

Results: In month 1, before intervention, CAM-ICU was performed 140 times in 2 days on 32 patients with an average CAM-ICU performed 2.2 times per patient/day. There were 3 concerning quotes for confusion during team rounds and 0 for delirium by nurses during team rounds. EHR review noted 7 patients had dementia, 2 had a positive CAM-ICU and 3 had a diagnosis of delirium. In month 2, after intervention, CAM-ICU was performed 163 times in 2 days on 35 patients with an average CAM-ICU performed 2.35 times per patient/ day. There were 6 concerning quotes regarding confusion and 1 regarding delirium by nurses during team rounds. EHR review noted 1 patient had dementia, 0 had a positive CAM-ICU and 0 patients with delirium diagnosis.

Conclusion: This quality improvement project using Justin-Time Teaching by a geriatrician during weekly rounds resulted in a modest increase in number of times CAMICU was performed, increased discussion of delirium during rounds, but no increase in delirium recognition using CAM-ICU. Areas for improvement include involving more physicians and nursing staff along with more structured delirium education.

\section{Stent Thrombosis: Regional Prevalence, Risk Factors, and Outcomes}

Andrew M. Ayers, Chi C. Cho, Robyn Shearer, M. Fuad Jan, Anjan Gupta

Aurora Cardiovascular Services, Aurora Health Care; Aurora Research Institute, Aurora Health Care

Background: Stent thrombosis is an infrequent but catastrophic complication of percutaneous coronary intervention (PCI). Many studies usually involve few stent thrombosis patients, generally less than 60 , given its prevalence. While dual antiplatelet therapy has decreased stent thrombosis significantly in the general population, there are still patients who present with occurrence and recurrence of stent thrombosis.

Purpose: We sought to define the prevalence of site-specific stent thrombosis in a larger cohort of patients by specific coronary territories and determine if this had an effect on cardiovascular outcomes. In addition we sought to elucidate the role of previous coronary artery bypass grafting (CABG) 